\title{
PSA-based all-optical multi-channel phase regenerator
}

\author{
F. Parmigiani, K. R. H. Bottrill, R. Slavík, D. J. Richardson, P. Petropoulos \\ Optoelectronics Research Centre, University of Southampton, Southampton, SO17 1BJ, UK, \\ frp@orc.soton.ac.uk
}

\begin{abstract}
We demonstrate simultaneous phase regeneration of six NRZ BPSK signals in a single nonlinear medium using FWM-based phase sensitive amplification. BER measurements confirm OSNR improvement and negligible cross-talk across all the regenerated channels, impaired with broadband phase noise.
\end{abstract}

\section{Introduction}

All-optical signal processing becomes competitive when advantage is taken of its ability to process very broad optical bandwidths. However, to maximise its benefit in telecommunication systems that are restricted by the limited bandwidth of electronics we must apply it concurrently to as many signals as possible ${ }^{1,2}$. Phase sensitive amplifiers (PSAs) based on four wave mixing (FWM) effects have been shown to be powerful tools for the processing of modern coherent signals, particularly when it comes to all-optical regeneration, with schemes capable of handling BPSK, QPSK and 16 QAM signal formats amongst others so far demonstrated ${ }^{3-5}$. However, most PSA experiments reported in the literature have supported a single channel with only a few attempts to simultaneously regenerate two or more channels in a single device $^{6}$, highlighting the challenges faced when attempting to achieve multi-channel operation. Non-degenerate configurations (in which a signal occupies two wavelength channels to simultaneously transmit the signal and its idler, have been employed for low noise figure amplification and have been shown to be capable of amplifying up to three 16 QAM channels using a single pump and a single nonlinear medium ${ }^{5}$. Although such schemes can amplify any modulation format, they sacrifice half of the transmission bandwidth (to transmit the idlers). In degenerate configurations, composed of two pumps and one wavelength channel to carry the information, regeneration of up to two channels was successfully demonstrated using two scalar PSAs, sharing one pump ${ }^{6}$. However, this work highlighted the limitations on the simultaneous regeneration of multiple signals imposed by the strong pump-topump (PtP) and pump-to-signal (PtS) FWM over a large bandwidth.

We have recently demonstrated a polarizationassisted (PA) PSA-based phase regenerator that uses two orthogonally polarized pumps (the signal is co-polarized with one of them), minimizing the PtP nonlinear interaction?. Another key advantage is that high phase sensitive extinction ratios, PSERs, (defined as the difference between the maximum PS- gain and the maximum PS- attenuation) can be achieved at modest pump powers, further reducing the undesired nonlinear interactions, the main cause of cross-talk in multi-channel operation. Here, we exploit these two key features together with the use of both propagation directions in the same nonlinear medium (here a highly nonlinear fibre (HNLF)) and a particular placement of the pump/signal wavelengths in the regenerator to avoid any PtP and PtS FWM products overlapping with the signals. This allows us to place six PA-PSAs, in the same non-linear medium and, thus, to simultaneously regenerate six WDM channels with minimum cross-talk among them, as bit error ratio (BER) measurements confirm.
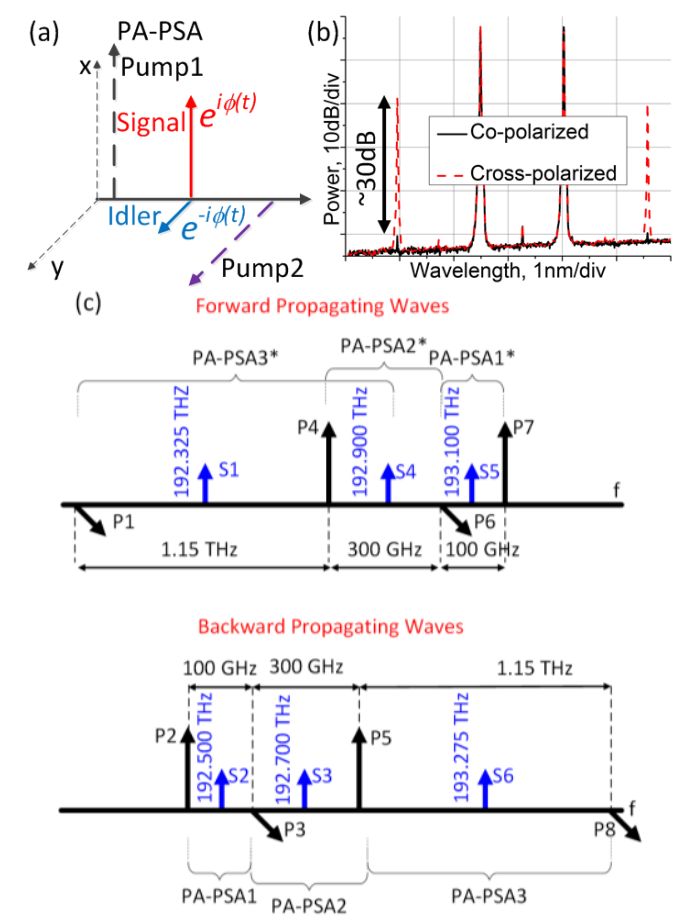

Fig. 1: a) PA-PSA configuration. b) Scalar and vector PtP FWM spectra. c) Pump and signal spectral locations for the forward (top) and backward (bottom) directions in the HNLF. 


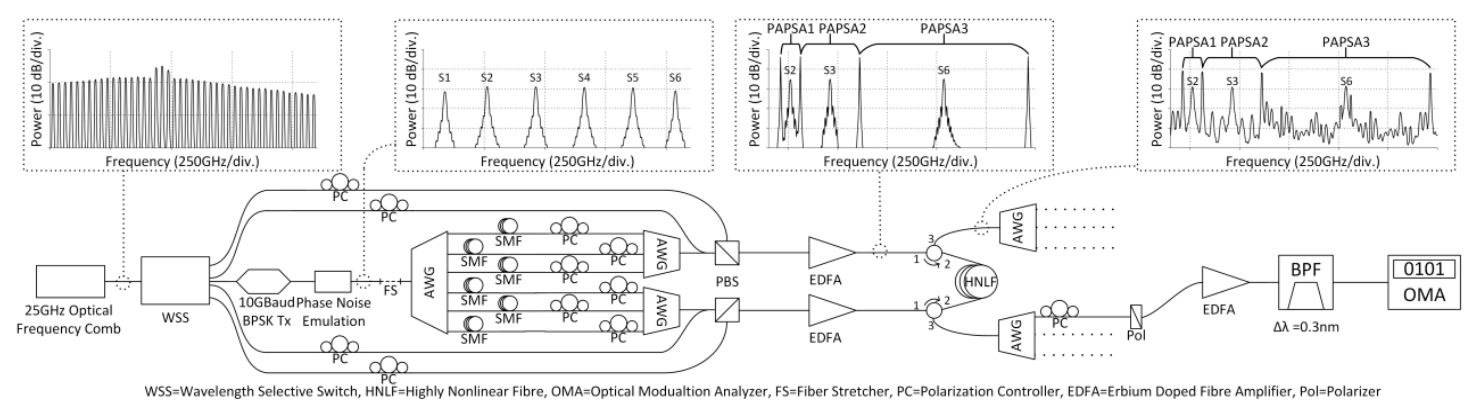

Fig. 2: Experimental setup. Inset figures: spectral traces at different points of the system.

Principle, Experimental Setup and Results

The PA-PSA is based on signal degenerate FWM in which the signal to be regenerated is co-polarized with one of two orthogonally polarized phase locked pumps, symmetrically placed in frequency about the signal. The degenerate phase-locked idler is generated at the same frequency, but orthogonal polarisation to the signal, thereby producing no PS interaction, see Fig.1 (a). However, PS operation may be achieved for virtually any pump power (provided sufficient OSNR) by passing the orthogonal signal-idler pair through a polarizer with an angle selected to equalize their relative powers ${ }^{7}$. Figure 1 (b) highlights the difference in terms of undesired PtP interactions for scalar and PA- PSAs. In this work, we profit from the following two key properties of PA-PSA as compared to scalar PSA: lower pump powers may be used and - more importantly for multichannel operation - PtP and PtS FWM are minimized, thereby requiring less spectral bandwidth for each PA-PSA operation. For multi-channel operation each PA-PSA shares a pump with the adjacent one, for a total of three PA-PSAs along each direction of the HNLF, as shown in Fig.1 (c).

The corresponding experimental setup is shown in Fig.2. A $25 \mathrm{GHz}$ optical frequency comb was used to guarantee signal and pump phase locking without the complexity of a black-box configuration ${ }^{3}$, see inset to Fig.2. The comb was fed to a wavelength selective switch (WSS) to select the lines constituting the pumps and signals and control their relative power. The six signal tones $(192.325,192.5,192.7,192.9$, 193.1, $193.275 \mathrm{THz}$ ), see inset to Fig.2, all propagating in a single fibre, were modulated using a 10 Gbaud non-return-to-zero (NRZ) BPSK signal. They were subsequently passed through a phase modulator which was used to apply a $70 \mathrm{kHz}$ sinusoidal dither (used for phase synchronization of the signal/pumps in the PAPSA) as well as broadband phase noise for noise emulation (achieved by detecting the amplified spontaneous emission noise of an EDFA). After de-correlating the data (through demultiplexing), they were re-combined using two arrayed waveguide gratings (AWGs) into two groups, combined with the corresponding pumps (see Fig.1 (c)) using an optical coupler and a polarization beam splitter (PBS) and launched into the HNLF via a circulator to allow bidirectional propagation. The waves at each input of the HNLF were amplified to about $18 \mathrm{dBm}$ per pump (with a signal to pump ratio of $\sim-11 \mathrm{~dB}$ ). The HNLF was a $302 \mathrm{~m}$-long germanium-silicate fibre with a nonlinear coefficient of $11.6(\mathrm{~W} \cdot \mathrm{km})^{-1}$, a dispersion slope of $0.018 \mathrm{ps} /\left(\mathrm{nm}^{2} \cdot \mathrm{km}\right)$, and an average zero dispersion wavelength of $1555 \mathrm{~nm}$. The insets to Fig.2 provide examples of spectra before and after the HNLF for one direction in the fibre. While some undesired FWM is observed at the output spectrum, no spurious component overlaps spectrally with the signals due to the careful choice of the frequency placement of the signals/pumps. Each individual channel was passed through an optical bandpass filter followed by a polarization controller (PC) and a polarizer and assessed using a pre-amplified coherent receiver and real time scope (optical modulation analyser, OMA). A small portion of the signal was tapped for tracking the signal phase using a feedback loop that drove a fibre stretcher (FS) to compensate for thermally- and acoustically-induced phase shifts. In the experiment, a single FS was placed in the signals' common path, while N FSs would be required for the simultaneous regeneration of $\mathrm{N}$ independent signals. The phase regenerator was assessed both in single and multi-channel operation when the incoming signals did not experience any noise (error vector magnitude $(E V M)$ of $~ 9.6 \%$ root mean square $(\mathrm{rms})$, magnitude error (ME) of $~ 7.6 \%$ rms and phase noise (PN) of $\sim 3.3^{\circ} \mathrm{rms}$ ) or under broadband phase noise (EVM of $\sim 41 \% \mathrm{rms}, \mathrm{ME}$ of $\sim 10 \%$ rms and $P N$ of $\sim 23^{\circ} \mathrm{rms}$ ). Figure 3 (a) illustrates an example of BER curves before and 


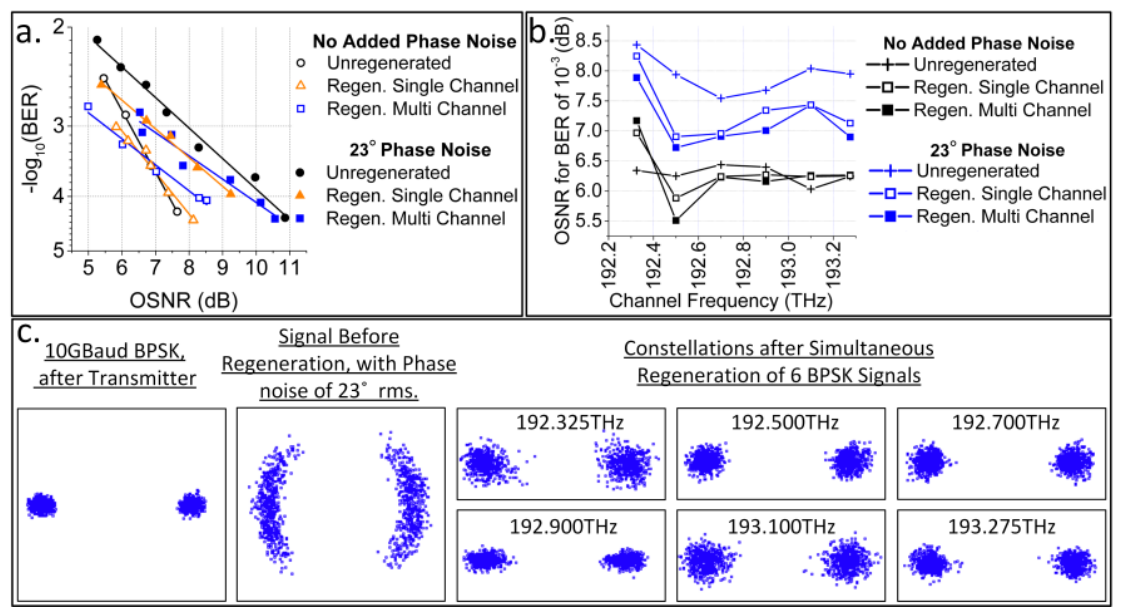

Fig. 3: a) Example of BER curves with and without added noise beforelafter the regenerator for single and multi-channel operation (presented for signal $192.5 \mathrm{THz}$ ). b) OSNRs for BER of $10^{-3}$ versus signal carrier frequency before/after the regenerator in single and multi-channel operation. c) Signal constellation diagrams at different points of the system.

after the regenerator, in single- and multichannel operation, with and without noise for the signal at $192.5 \mathrm{THz}$. Similar performance was obtained for all the channels, as shown in Fig. 3 (b), which reports the OSNRs at a BER of $10^{-3}$ for all of the signals. Negligible penalty was observed when no noise was added to the various signals both in single and multi-channel operation. When noise was added to the signal, the performance of the regeneration system was very similar for single- and multi-channel operation, highlighting the absence of channel cross-talk. The maximum/minimum OSNR improvement for the multi-channel operation was $1.2 \mathrm{~dB} / 0.55 \mathrm{~dB}$, achieved for signals at 192.325 THz/ 192.5 THz, respectively. Optimum performance was achieved for the central signals, most likely due to the better OSNR of the corresponding pumps at these wavelengths, see comb source in Fig.2. However, all the regenerated signals showed a performance improvement as compared to the noisy input signals. Figure 3 (c) shows the constellation diagrams of a typical signal, before/after noise addition, and of all the signals after regeneration in the presence of broadband noise.

\section{Conclusions}

We have reported the simultaneous phase regeneration of six BPSK channels using FWMbased PA- PSAs. Due to the orthogonality of the pumps, the low pump power used and the choice of the frequency grid for the signals/pumps location, no BER penalty is observed between multi- and single-channel operations, highlighting the absence of any channel cross-talk. In both configurations, up to $1.2 \mathrm{~dB}$ OSNR improvement for a BER of $10^{-3}$ was achieved as compared to the back-to-back when the signals were contaminated with broadband phase noise. While the regenerator was characterized at 10 Gbaud due to the components available, it is worth noting that the scheme can be easily scaled to much higher baud rates simply by increasing the frequency spacing between the pumps. We believe that the technique is extendable to accommodate an even greater number of channels if the signals can be located in broader spectral grids and a more dispersive HNLF is used.

\section{Acknowledgements}

Research sponsored by EPSRC grant EP/101196X, The Photonics Hyperhighway. Dr F. Parmigiani is a Royal Academy of Engineering/EPSRC Research Fellow.

\section{References}

[1] P.G. Patki, M. Vasilyev, T.I. Lakoba, "Multichannel alloptical regeneration", in Photonics Society Summer Topical Meeting Series (2010), pp. 172-173.

[2] F. Parmigiani, et al., "Progress in multichannel all-optical regeneration based on fiber technology", IEEE Sel. Top. Quantum Electron. 18(2), 689-699 (2012).

[3] R. Slavik, et al., "All-optical phase and amplitude regenerator for next-generation telecommunications systems," Nat. Phot., vol. 4, 690-695 (2010).

[4] J. Kakande, et al., "Multilevel quantization of optical phase in a novel coherent parametric mixer architecture," Nat. Photonics 5(12), 748-752 (2011).

[5] T. Umeki, et al., "First demonstration of high- order QAM signal amplification in PPLN-based phase sensitive amplifier", Opt. Express 22(3), 2473-2482 (2014).

[6] S. Sygletos, et al., "A practical phase sensitive amplification scheme for two channel phase regeneration", Opt. Express 19, B938-B945 (2011).

[7] F. Parmigiani, et al., "Polarization-Assisted PhaseSensitive Processor", J. of Lightwave Technol., 33(6), 1166-1174 (2015). 